\title{
On Convergence and Solvability of an Elliptic Equation by Finite Difference Method
}

\author{
A.J.A. RAMOS \\ Received on September 1, 2016 / Accepted on December 9, 2016
}

\begin{abstract}
In this article we deal with questions of convergence, existence and uniqueness of the numerical solutions for a discretized elliptic problem by using finite difference method. In order to show existence and uniqueness of numerical solutions we will use a suitable variational setup (at discrete level) to guarantee the existence of a numerical solution by means of a finite difference method.
\end{abstract}

Keywords: elliptic equation, finite difference, solvability of numerical solutions.

\section{INTRODUCTION}

It is well known that the general model

$$
\left\{\begin{aligned}
-\Delta u+q(x) u & =f(x) & & \text { in } \Omega, \\
u & =0 & & \text { on } \quad \partial \Omega
\end{aligned}\right.
$$

where $q, f \in C(\Omega)$ has been vastly studied in the last years. See, for example, $[1,2,3,4]$ and the references therein.

Here, $\Omega \subset \mathbb{R}^{N}, N \geq 1$ is a bounded smooth domain and $f: \Omega \rightarrow \mathbb{R}$ is a given function has relevant physical motivation as, for instance, stationary solutions of heat and wave equations, population models and geometric models and so on. Besides of this we have several nonlocal models like

$$
\left\{\begin{array}{rll}
-M(B(u)) \Delta u+q(x) u & =f(x) & \text { in } \Omega, \\
u & =0 & \text { on } \partial \Omega,
\end{array}\right.
$$

where $M$ is a given function and $B$ is an integral operator, is also of paramount importance in the modeling of several phenomena. In a forthcoming paper we will attack this last problem.

Let us recall some known results on existence and uniqueness of solutions to the problem (1.1). This is important for aims that we have in mind.

Laboratório de Inovação Interdisciplinar - LabX, Faculdade de Ciências, Universidade Federal do Pará, Campus Universitário de Salinópolis, Rua Raimundo Santana Cruz, S/N, 68721-000, Salinópolis, PA, Brasil. E-mail: ramos@ufpa.br 
The problem (1.1) is so-called the homogeneous Dirichlet problem. Generally speaking, the Dirichlet problem consists in coupling a differential equation with a boundary condition that specifies the values of the unknown function on the boundary of $\Omega$; one says that the Dirichlet condition is homogeneous if the unknown is required to be zero on $\partial \Omega$.

Let us take $q \in L^{\infty}(\Omega)$ and $f \in L^{2}(\Omega)$. It is well know that a weak solution of problem (1.1) is a function $u \in H_{0}^{1}(\Omega)$ such that

$$
\int_{\Omega} \nabla u \cdot \nabla v d x+\int_{\Omega} q(x) u v d x=\int_{\Omega} f(x) v d x, \forall v \in H_{0}^{1}(\Omega) .
$$

As consequence of this variational formulation, we define the functional $J: H_{0}^{1}(\Omega) \rightarrow \mathbb{R}$ given by

$$
J(u):=\frac{1}{2} \int_{\Omega}|\nabla u|^{2} d x+\frac{1}{2} \int_{\Omega} q(x)|u|^{2} d x-\int_{\Omega} f(x) v d x .
$$

This functional is often called the energy functional associated to problem (1.1) in its importance relies from applications, where $J$ is likely to represent an energy of some sort. Moreover, it is well known that the functional $J$ is differentiable on $H_{0}^{1}(\Omega)$ and its derivative is given by

$$
\begin{aligned}
J^{\prime}(u) v & =\int_{\Omega} \nabla u \cdot \nabla v d x+\int_{\Omega} q(x) u v d x \\
& -\int_{\Omega} f(x) v d x, \quad \forall v \in H_{0}^{1}(\Omega) .
\end{aligned}
$$

Therefore, comparing (1.3) and (1.5), one sees that the functions $u$ is a weak solution of problem (1.1) if and only if $u$ is a critical point of the functional $J$. Furthermore it is shown that $J$ is continuous, coercive and strictly convex which ensures the existence of global minimum point and consequently existence and uniqueness of solution to the problem (1.1).

\subsection{Numerical setting and statement of the results}

In this work, we are concerned with a discrete version of the problem (1.1) at numerical setting of the finite differences. Here, we take $\Omega \subset \mathbb{R}^{2}$.

More precisely, we consider the following numerical scheme:

$$
\left\{\begin{array}{rlrl}
\mathcal{L}_{d} u_{i, j} & =f_{i, j}, & 0 \leq i \leq N, 0 \leq j \leq M, \text { in } \Omega_{d} \\
u_{i, 0}=u_{0, j} & =0, & & 0 \leq i \leq N+1,0 \leq j \leq M+1
\end{array}\right.
$$

where we are assuming that

$$
\mathcal{L}_{d} u_{i, j}:=-\Delta_{d} u_{i, j}+q_{i, j} u_{i, j}
$$

where

$$
\Delta_{d} u_{i, j}:=\frac{u_{i+1, j}-2 u_{i, j}+u_{i-1, j}}{\Delta x^{2}}+\frac{u_{i, j+1}-2 u_{i, j}+u_{i, j-1}}{\Delta y^{2}} .
$$


The numerical operator (1.8) is the finite difference operator centered for the $2^{\text {nd }}$ order derivative, known as five points formula. Here, the discrete solutions $u_{i, j}$ and $v_{i, j}$ are approximations to $u\left(x_{i}, y_{j}\right)$ and $v\left(x_{i}, y_{j}\right)$ at the mesh points $\left(x_{i}, y_{j}\right)$, respectively.

Moreover, $q_{i, j} \in l^{\infty}\left(\Omega_{d}\right)$ and $f_{i, j} \in l_{0}^{2}\left(\Omega_{d}\right)$. The space $l_{0}^{2}\left(\Omega_{d}\right)$ is defined as

$$
l_{0}^{2}\left(\Omega_{d}\right)=\left\{u_{i, j}: \Omega_{d} \rightarrow \mathbb{R} ;\left(\sum_{i=0}^{N} \sum_{j=0}^{M}\left|u_{i, j}\right|^{2}\right)^{1 / 2}<\infty\right\},
$$

satisfying $u_{i, 0}=u_{0, j}=0$, for all $0 \leq i \leq N+1$ and $0 \leq j \leq M+1$. This discrete space is equipped with the inner product and the norm given, respectively, by

$$
\begin{aligned}
\left(u_{i, j}, v_{i, j}\right)_{l^{2}\left(\Omega_{d}\right)} & =\Delta x \Delta y \sum_{j=0}^{M} \sum_{i=0}^{N} u_{i, j} v_{i, j}, \\
\left|u_{i, j}\right|_{l^{2}\left(\Omega_{d}\right)} & =\left(u_{i, j}, u_{i, j}\right)_{l^{2}\left(\Omega_{d}\right)}^{1 / 2} .
\end{aligned}
$$

Moreover, we defined other inner product as

$$
\begin{aligned}
\left(\left(\nabla u_{i, j}, \nabla v_{i, j}\right)\right) & =\Delta x \Delta y \sum_{i=0}^{N} \sum_{j=0}^{M} \nabla_{x} u_{i, j} \cdot \nabla_{x} v_{i, j} \\
& +\Delta x \Delta y \sum_{i=0}^{N} \sum_{j=0}^{M} \nabla_{y} u_{i, j} \cdot \nabla_{y} v_{i, j},
\end{aligned}
$$

and norm as

$$
\left\|\nabla u_{i, j}\right\|=\left(\left(\nabla u_{i, j}, \nabla u_{i, j}\right)\right)^{1 / 2}
$$

In (1.12), we have used the numerical operators given by

$$
\nabla_{x} u_{i, j}:=\frac{u_{i+1, j}-u_{i, j}}{\Delta x}, \quad \nabla_{y} u_{i, j}:=\frac{u_{i, j+1}-u_{i, j}}{\Delta y} .
$$

Moreover, the space $l_{0}^{\infty}\left(\Omega_{d}\right)$ is defined as being the discrete space of the real bounded sequences

$$
l_{0}^{\infty}\left(\Omega_{d}\right)=\left\{u_{i, j}: \Omega_{d} \rightarrow \mathbb{R} ; \sup _{0 \leq i, j \leq N, M}\left|u_{i, j}\right|<\infty\right\},
$$

obeying $u_{i, 0}=u_{0, j}=0$, for all $0 \leq i \leq N+1,0 \leq j \leq M+1$. This space is equipped with the norm

$$
\left\|u_{i, j}\right\|_{\infty}=\sup _{0 \leq i, j \leq N, M}\left|u_{i, j}\right|
$$


The discrete domain $\Omega_{d}$ is given by a discretization of the rectangle $\left[0, L_{1}\right] \times\left[0, L_{2}\right]$. We consider a discretization of the intervals $\left[0, L_{1}\right]$ and $\left[0, L_{2}\right]$ given by

$$
\begin{aligned}
& 0=x_{0}<x_{1}<\ldots<x_{i}=i \Delta x<\ldots<x_{N}<x_{N+1}=L_{1}, \\
& 0=y_{0}<y_{1}<\ldots<y_{j}=j \Delta y<\ldots<y_{M}<y_{M+1}=L_{2},
\end{aligned}
$$

where $\Delta x=L_{1} /(N+1), \Delta y=L_{2} /(M+1)$ and $N, M \in \mathbb{N}$. Hence,

$$
\Omega_{d}=\bigcup_{i, j=0}^{N, M}\left(x_{i}, x_{i+1}\right) \times\left(y_{j}, y_{j+1}\right) \quad \text { and } \lim _{\Delta x, \Delta y \rightarrow 0} \Omega_{d}=\left(0, L_{1}\right) \times\left(0, L_{2}\right) .
$$

The main results of this paper are as follows:

Theorem 1.1. Let $\Omega=\left(0, L_{1}\right) \times\left(0, L_{1}\right)$ and $u \in C^{4}(\bar{\Omega})$ be a classical solution of the Dirichlet problem

$$
\left\{\begin{array}{rlrl}
-\Delta u+q(x) u & =f(x), & & \text { in } \Omega \\
u & =0, & \text { on } \partial \Omega
\end{array}\right.
$$

where $\Omega_{d} \subset \bar{\Omega}$ is a discrete domain and $u_{i, j}$ a corresponding solution of the discretized problem

$$
\left\{\begin{aligned}
\mathcal{L}_{d} u_{i, j} & =f_{i, j}, & & 0 \leq i \leq N, 0 \leq j \leq M, \text { in } \Omega_{d} \\
u_{i, 0}=u_{0, j} & =0, & & 0 \leq i \leq N+1,0 \leq j \leq M+1 .
\end{aligned}\right.
$$

Then, there exists a positive constant $C$ independent of $u$ satisfying the following estimate:

$$
\left\|u\left(x_{i}, y_{j}\right)-u_{i, j}\right\|_{\infty} \leq C\left\|D^{4} u\right\|_{L^{\infty}(\Omega)}\left(\Delta x^{2}+\Delta y^{2}\right)
$$

Theorem 1.2. Let $\Omega_{d} \subset\left[0, L_{1}\right] \times\left[0, L_{2}\right]$ be a discrete domain. Assuming $q_{i, j} \in l^{\infty}\left(\Omega_{d}\right)$ and $f_{i, j} \in l_{0}^{2}\left(\Omega_{d}\right)$, then there exists only one solution of the discrete problem (1.17).

We highlight some observations on numerical convergence. When we deal with parabolic problems like

$$
\frac{u_{i, j}^{n+1}-u_{i, j}^{n-1}}{2 \Delta t}-\frac{u_{i+1, j}^{n}-2 u_{i, j}^{n}+u_{i-1, j}^{n}}{\Delta x^{2}}-\frac{u_{i, j+1}^{n}-2 u_{i, j}^{n}+u_{i, j-1}^{n}}{\Delta y^{2}}=f_{i, j},
$$

the convergence analysis is performed through the condition CFL (Courant-Friedrichs-Lewy), where the numerical stability is verified accordingly a Von Neumann condition (see [5]). It is clear that, in the elliptic case, we are not able to use the same arguments. Because of this, we use certain tools typical of elliptic equations, like Discrete Maximum Principle (see Section 10.3 of Thomas [6]) in order to guarantee the a priori estimate and, consequently, to reach in the numerical convergence of the Poisson problem

$$
-\frac{u_{i+1, j}-2 u_{i, j}+u_{i-1, j}}{\Delta x^{2}}-\frac{u_{i, j+1}-2 u_{i, j}+u_{i, j-1}}{\Delta y^{2}}=f_{i, j} .
$$


Still concerning with the Poisson problem, the proof of the existence and uniqueness of the discrete solution, given in $[7,6,8]$, consists in showing that the matrix

$$
A=\left(\begin{array}{cccccc}
B & -\frac{1}{\Delta y^{2}} I & & & & \\
-\frac{1}{\Delta y^{2}} I & B & -\frac{1}{\Delta y^{2}} I & & & \\
& -\frac{1}{\Delta y^{2}} I & \ddots & \ddots & & \\
& & \ddots & \ddots & -\frac{1}{\Delta y^{2}} I & \\
& & & -\frac{1}{\Delta y^{2}} I & B & -\frac{1}{\Delta y^{2}} I \\
& & & & -\frac{1}{\Delta y^{2}} I & B
\end{array}\right)_{(M-1) \times(M-1)}
$$

associated to the numerical problem, where $I$ is the $(N-1) \times(N-1)$ identity matrix and $B$ uma matriz tridiagonal $(N-1) \times(N-1)$, is a positive definite symmetric matrix and in order to obtain invertibility we should have.

Here we use a technique which is not usual in this kind of problem. Indeed, following ideas similar to those used in continuous case, we consider a variational approach at discrete setting. In this way we consider an discrete functional in such a way their critical points are weak solutions of our problem.

Our approach is this work is twofold. Firstly, we focus on the existence of an priori estimate in order to guarantee the convergence of the numerical solution. Secondly, we build a discrete functional in order to prove the existence and uniqueness of solution for the discrete problem (1.6).

\subsection{Outline of the paper}

The plan of this paper is as follows: in Section 2 we establish the convergence of numerical solutions. In particular, we proved a discrete version to the Maximum Principle. In Section 3 we treated a variational formulation in numerical setting and in Section 4 we prove our main results. Finally, in Section 5, we proved with numerical experiments some of these results.

\section{CONVERGENCE OF THE DISCRETE SOLUTION}

In this section, we prove a Discrete Maximum Principle playing an important role in the proof of the a priori estimate for solutions of the problem (1.6). Moreover, we prove a result on estimative a priori of the discrete solution of our problem.

Theorem 2.1 (Discrete Maximum Principle). Let

$$
\mathcal{L}_{d} u_{i, j}:=-\Delta_{d} u_{i, j}+q_{i, j} u_{i, j} \leq(\geq) 0, \quad \text { in } \quad \Omega_{d} .
$$

Then the maximum (minimum) value $u_{i, j}$ is reached in $\partial \Omega_{d} \subset \bar{\Omega}_{d}$. 
Proof. To proof our assertive, we consider that the maximum of $u_{i, j}$ is not attained in $\partial \Omega_{d}$. First we note that $\mathcal{L}_{d} u_{i, j} \leq 0$ is equivalent with

$$
\begin{aligned}
\left(\frac{1}{\Delta x^{2}}+\frac{1}{\Delta y^{2}}+\frac{q_{i, j}}{2}\right) u_{i, j} & \leq \frac{1}{2 \Delta x^{2}}\left(u_{i+1, j}+u_{i-1, j}\right) \\
& +\frac{1}{2 \Delta y^{2}}\left(u_{i, j+1}+u_{i, j-1}\right)
\end{aligned}
$$

for all $0 \leq i \leq N, 0 \leq j \leq M$.

Suppose that $u_{i, j}$ is the local maximum. Then, for all indices $i \neq 0, N+1, j \neq 0, M+1$ we have

$$
u_{i, j} \geq u_{i+1, j}, u_{i, j} \geq u_{i-1, j}, u_{i, j} \geq u_{i, j+1} \text { and } u_{i, j} \geq u_{i, j-1} .
$$

Taking into account these inequalities, we can rewritten (2.2) as

$$
\begin{aligned}
\left(\frac{1}{\Delta x^{2}}+\frac{1}{\Delta y^{2}}+\frac{q_{i, j}}{2}\right) u_{i, j} & \leq \frac{1}{2}\left[\frac{1}{\Delta x^{2}} u_{i+1, j}+\frac{1}{\Delta x^{2}} u_{i, j}+\frac{2}{\Delta y^{2}} u_{i, j}\right] \\
& \leq\left(\frac{1}{\Delta x^{2}}+\frac{1}{\Delta y^{2}}\right) u_{i, j},
\end{aligned}
$$

from where we have, since $q_{i, j} \geq 0$ for all $0 \leq i \leq N, 0 \leq j \leq M$,

$$
\left(\frac{1}{\Delta x^{2}}+\frac{1}{\Delta y^{2}}\right) u_{i, j} \leq \frac{1}{2}\left[\frac{1}{\Delta x^{2}} u_{i+1, j}+\frac{1}{\Delta x^{2}} u_{i, j}+\frac{2}{\Delta y^{2}} u_{i, j}\right] \leq\left(\frac{1}{\Delta x^{2}}+\frac{1}{\Delta y^{2}}\right) u_{i, j},
$$

and then

$$
\frac{1}{\Delta x^{2}} u_{i, j}=\frac{1}{2}\left[\frac{1}{\Delta x^{2}} u_{i+1, j}+\frac{1}{\Delta x^{2}} u_{i, j}\right]
$$

and, consequently,

$$
u_{i+1, j}=u_{i, j}, \forall i \neq 0, N+1, j \neq 0, M+1 .
$$

Reasoning in the same way, we get

$$
u_{i, j}=u_{i+1, j}=u_{i-1, j}=u_{i, j+1}=u_{i, j-1}, \quad \forall i \neq 0, N+1, j \neq 0, M+1 .
$$

This shows that $u_{i, j}$ is a constant function which obviously is a contradiction and therefore the maximum is attained on the boundary. The proof of the minimum principle is performed in the same way.

Now, without loss of generality, we consider $L_{1}=L_{2}=1$ and we will work in the unit square $\Omega=(0,1) \times(0,1)$. We will use the discrete sup norm for functions defined in the discretized domain $\bar{\Omega}_{d}$, that is,

$$
\left\|u_{i, j}\right\|_{\infty}=\max _{i, j \in \mathbb{N}}\left|u_{i, j}\right|, \forall i, j
$$


At first we obtain a priori estimate, which may be seen as a discrete regularity result, for solutions of a homogeneous discrete Dirichlet problem.

Theorem 2.2 (A Priori Estimate). Let $u_{i, j}$ be a solution of discrete system (1.6). Then, we have

$$
\left\|u_{i, j}\right\|_{\infty} \leq\left\|\mathcal{L}_{d} u_{i, j}\right\|_{\infty}, \forall i, j
$$

where $\mathcal{L}_{d} u_{i, j}=-\Delta_{d} u_{i, j}+q_{i, j} u_{i, j}$ and $0 \leq q_{i, j} \in l^{\infty}\left(\Omega_{d}\right)$.

Proof. Let us consider the discrete function $w_{i, j}$ defined as

$$
w_{i, j}:=\frac{1}{4}\left[\left(x_{i}-\frac{1}{2}\right)^{2}+\left(y_{j}-\frac{1}{2}\right)^{2}\right] \geq 0, \forall i, j,
$$

with $\left\|q_{i, j}\right\|_{\infty}<\left\|w_{i, j}\right\|_{\infty}^{-1}$. For this function we statement that

$$
-1 \leq \mathcal{L}_{d} w_{i, j} \leq-1+\frac{\left\|q_{i, j}\right\|_{\infty}}{8}, \forall i, j .
$$

Indeed, we have

$$
\begin{aligned}
& \mathcal{L}_{d} w_{i, j}=-\frac{1}{4} \frac{\left(x_{i+1}-\frac{1}{2}\right)^{2}+\left(y_{j}-\frac{1}{2}\right)^{2}-2\left(x_{i}-\frac{1}{2}\right)^{2}-2\left(y_{j}-\frac{1}{2}\right)^{2}}{\Delta x^{2}} \\
& -\frac{1}{4} \frac{\left(x_{i-1}-\frac{1}{2}\right)^{2}+\left(y_{j}-\frac{1}{2}\right)^{2}}{\Delta x^{2}} \\
& -\frac{1}{4} \frac{\left(x_{i}-\frac{1}{2}\right)^{2}+\left(y_{j+1}-\frac{1}{2}\right)^{2}-2\left(x_{i}-\frac{1}{2}\right)^{2}-2\left(y_{j}-\frac{1}{2}\right)^{2}}{\Delta x^{2}} \\
& -\frac{1}{4} \frac{\left(x_{i}-\frac{1}{2}\right)^{2}+\left(y_{j-1}-\frac{1}{2}\right)^{2}}{\Delta x^{2}} \\
& +\frac{q_{i, j}}{4}\left[\left(x_{i}-\frac{1}{2}\right)^{2}+\left(y_{j}-\frac{1}{2}\right)^{2}\right] \text {. } \\
& \mathcal{L}_{d} w_{i, j}=-\frac{1}{4} \frac{\left(x_{i+1}-\frac{1}{2}\right)^{2}-2\left(x_{i}-\frac{1}{2}\right)^{2}+\left(x_{i-1}-\frac{1}{2}\right)^{2}}{\Delta x^{2}} \\
& +\frac{1}{4} \frac{\left(y_{j+1}-\frac{1}{2}\right)^{2}-2\left(y_{j}-\frac{1}{2}\right)^{2}+\left(y_{j-1}-\frac{1}{2}\right)^{2}}{\Delta y^{2}} \\
& +\frac{q_{i, j}}{4}\left[\left(x_{i}-\frac{1}{2}\right)^{2}+\left(y_{j}-\frac{1}{2}\right)^{2}\right] \\
& \mathcal{L}_{d} w_{i, j}=-\frac{1}{4} \frac{\left(x_{i}+\Delta x-\frac{1}{2}\right)^{2}-2\left(x_{i}-\frac{1}{2}\right)^{2}+\left(x_{i}-\Delta x-\frac{1}{2}\right)^{2}}{\Delta x^{2}} \\
& -\frac{1}{4} \frac{\left(y_{j}+\Delta y-\frac{1}{2}\right)^{2}-2\left(y_{j}-\frac{1}{2}\right)^{2}+\left(y_{j}-\Delta y-\frac{1}{2}\right)^{2}}{\Delta y^{2}} \\
& +\frac{q_{i, j}}{4}\left[\left(x_{i}-\frac{1}{2}\right)^{2}+\left(y_{j}-\frac{1}{2}\right)^{2}\right] \text {. }
\end{aligned}
$$

Tend. Mat. Apl. Comput., 18, N. 1 (2017) 
Consequently, we obtain

$$
\begin{aligned}
-1 \leq \mathcal{L}_{d} w_{i, j} & =-1+\frac{q_{i, j}}{4}\left[\left(x_{i}-\frac{1}{2}\right)^{2}+\left(y_{j}-\frac{1}{2}\right)^{2}\right] \\
& \leq-1+\frac{\left\|q_{i, j}\right\|_{\infty}}{4}\left[\max _{0 \leq x_{i} \leq 1}\left(x_{i}-\frac{1}{2}\right)^{2}+\max _{0 \leq y_{j} \leq 1}\left(y_{j}-\frac{1}{2}\right)^{2}\right] \\
& =-1+\frac{\left\|q_{i, j}\right\|_{\infty}}{8} .
\end{aligned}
$$

Now, let us assume that $\left\|q_{i, j}\right\|_{\infty}=7<\left\|w_{i, j}\right\|_{\infty}^{-1}$, from where we obtain

$$
-1 \leq \mathcal{L}_{d} w_{i, j} \leq-\frac{1}{8}
$$

and then we can define

$$
\mathcal{L}_{d} w_{i, j}=-\xi_{i, j} \in \mathcal{I}, \quad \xi_{i, j} \geq 0,
$$

for $0 \leq i \leq N+1,0 \leq j \leq M+1$ and $\mathcal{I}=[-1,-1 / 8]$. Moreover, we define the discrete functional

$$
\begin{aligned}
g_{i, j}^{ \pm}: l_{0}^{2}\left(\Omega_{d}\right) \times l_{0}^{2}\left(\Omega_{d}\right) & \rightarrow \mathbb{R} \\
\left(u_{i, j}, w_{i, j}\right) & \mapsto g_{i, j}^{ \pm}=u_{i, j} \pm 8\left\|\mathcal{L}_{d} u_{i, j}\right\|_{\infty} w_{i, j}
\end{aligned}
$$

to obtain

$$
\begin{aligned}
\mathcal{L}_{d} g_{i, j}^{-} & =\mathcal{L}_{d}\left(u_{i, j}-8\left\|\mathcal{L}_{d} u_{i, j}\right\|_{\infty} w_{i, j}\right)=\mathcal{L}_{d} u_{i, j}-8\left\|\mathcal{L}_{d} u_{i, j}\right\|_{\infty} \mathcal{L}_{d} w_{i, j} \\
& =\mathcal{L}_{d} u_{i, j}+8 \xi_{i, j}\left\|\mathcal{L}_{d} u_{i, j}\right\|_{\infty} \geq 0
\end{aligned}
$$

form where by using the Maximum Principle that the function $g_{i, j}^{-}$attains its minimum value at the boundary. In view of this we get

$$
u_{i, j} \geq g_{i, j}^{-} \geq-8\left\|\mathcal{L}_{d} u_{i, j}\right\|_{\infty} \max _{\partial \Omega_{d}} w_{i, j},
$$

and from definition of $w_{i, j}$ em (2.6) we obtain $\max _{\partial \Omega_{d}} w_{i, j}=\frac{1}{8}$ and then

$$
u_{i, j} \geq-\left\|\mathcal{L}_{d} u_{i, j}\right\|_{\infty}, 1 \leq i \leq N, \quad 1 \leq j \leq M
$$

In the same way, it follows that the function $g_{i, j}^{+}$attains its maximum at the boundary, that is,

$$
u_{i, j} \leq\left\|\mathcal{L}_{d} u_{i, j}\right\|_{\infty}, 1 \leq i \leq N, \quad 1 \leq j \leq M
$$

Combining the inequalities (2.8) and (2.9) it follows that

$$
\left|u_{i, j}\right| \leq\left\|\mathcal{L}_{d} u_{i, j}\right\|_{\infty}, \forall 1 \leq i \leq N, \quad \forall 1 \leq j \leq M,
$$

and we conclude the proof. 


\section{VARIATIONAL FORMULATION AND THE SPECTRUM AT DISCRETE SETTING}

In this section, we are concerned with discrete solutions of the discrete problem (1.6) satisfying a summable identity. In this way we have the following result:

Theorem 3.1. Let us consider $q_{i, j} \in l^{\infty}\left(\Omega_{d}\right)$ and $f_{i, j} \in l^{2}\left(\Omega_{d}\right)$. The discrete solution of the problem (1.6) obeys the following identity:

$$
\begin{array}{r}
\Delta x \Delta y \sum_{i=0}^{N} \sum_{j=0}^{M}\left(\nabla_{x} u_{i, j} \cdot \nabla_{x} v_{i, j}\right)+\Delta x \Delta y \sum_{i=0}^{N} \sum_{j=0}^{M}\left(\nabla_{y} u_{i, j} \cdot \nabla_{y} v_{i, j}\right) \\
+\Delta x \Delta y \sum_{i=0}^{N} \sum_{j=0}^{M} q_{i, j} u_{i, j} v_{i, j}=\Delta x \Delta y \sum_{i=0}^{N} \sum_{j=0}^{M} f_{i, j} v_{i, j}, \forall v_{i, j} \in l_{0}^{2}\left(\Omega_{d}\right) .
\end{array}
$$

Proof. Firstly, multiplying both sides of the equation (1.6) by $v_{i, j} \in l_{0}^{2}\left(\Omega_{d}\right)$ and summing up for $1 \leq i \leq N$ and $1 \leq j \leq M$ we obtain

$$
\begin{aligned}
& -\Delta x \Delta y \sum_{i=1}^{N} \sum_{j=1}^{M}\left(\frac{u_{i+1, j}-u_{i, j}}{\Delta x^{2}}\right) v_{i, j}-\Delta x \Delta y \sum_{i=1}^{N} \sum_{j=1}^{M}\left(\frac{u_{i-1, j}-u_{i, j}}{\Delta x^{2}}\right) v_{i, j} \\
& -\Delta x \Delta y \sum_{i=1}^{N} \sum_{j=1}^{M}\left(\frac{u_{i, j+1}-u_{i, j}}{\Delta y^{2}}\right) v_{i, j}-\Delta x \Delta y \sum_{i=1}^{N} \sum_{j=1}^{M}\left(\frac{u_{i, j-1}-u_{i, j}}{\Delta y^{2}}\right) v_{i, j} \\
& +\quad \Delta x \Delta y \sum_{i=1}^{N} \sum_{j=1}^{M} q_{i, j} u_{i, j} v_{i, j}=\Delta x \Delta y \sum_{i=1}^{N} \sum_{j=1}^{M} f_{i, j} v_{i, j} .
\end{aligned}
$$

For appropriate algebraic manipulations on boundary terms, we get

$$
\begin{aligned}
& -\Delta x \Delta y \sum_{i=0}^{N} \sum_{j=0}^{M}\left(\frac{u_{i+1, j}-u_{i, j}}{\Delta x^{2}}\right) v_{i, j}-\Delta x \Delta y \sum_{i=0}^{N} \sum_{j=0}^{M}\left(\frac{u_{i, j}-u_{i+1, j}}{\Delta x^{2}}\right) v_{i+1, j} \\
& -\Delta x \Delta y \sum_{i=0}^{N} \sum_{j=0}^{M}\left(\frac{u_{i, j+1}-u_{i, j}}{\Delta y^{2}}\right) v_{i, j}-\Delta x \Delta y \sum_{i=0}^{N} \sum_{j=0}^{M}\left(\frac{u_{i, j}-u_{i, j+1}}{\Delta y^{2}}\right) v_{i, j+1} \\
& +\Delta x \Delta y \sum_{i=0}^{N} \sum_{j=0}^{M} q_{i, j} u_{i, j} v_{i, j}=\Delta x \Delta y \sum_{i=0}^{N} \sum_{j=0}^{M} f_{i, j} v_{i, j},
\end{aligned}
$$


and then

$$
\begin{gathered}
\Delta x \Delta y \sum_{i=0}^{N} \sum_{j=0}^{M}\left(\frac{u_{i+1, j}-u_{i, j}}{\Delta x} \frac{v_{i+1, j}-v_{i, j}}{\Delta x}\right)+\Delta x \Delta y \sum_{i=0}^{N} \sum_{j=0}^{M}\left(\frac{u_{i, j+1}-u_{i, j}}{\Delta y} \frac{v_{i, j+1}-v_{i, j}}{\Delta y}\right) \\
+\Delta x \Delta y \sum_{i=0}^{N} \sum_{j=0}^{M} q_{i, j} u_{i, j} v_{i, j}=\Delta x \Delta y \sum_{i=0}^{N} \sum_{j=0}^{M} f_{i, j} v_{i, j} .
\end{gathered}
$$

Therefore, we obtain a discrete formulation in finite differences consisting in the following: to find $u_{i, j} \in l_{0}^{2}\left(\Omega_{d}\right)$ such

$$
\begin{aligned}
& \Delta x \Delta y \sum_{i=0}^{N} \sum_{j=0}^{M}\left(\nabla_{x} u_{i, j} \cdot \nabla_{x} v_{i, j}\right)+\Delta x \Delta y \sum_{i=0}^{N} \sum_{j=0}^{M}\left(\nabla_{y} u_{i, j} \cdot \nabla_{y} v_{i, j}\right) \\
& +\Delta x \Delta y \sum_{i=0}^{N} \sum_{j=0}^{M} q_{i, j} u_{i, j} v_{i, j}=\Delta x \Delta y \sum_{i=0}^{N} \sum_{j=0}^{M} f_{i, j} v_{i, j}, \forall v_{i, j} \in l_{0}^{2}\left(\Omega_{d}\right),
\end{aligned}
$$

and then we conclude the proof.

Invoking what was done above we define in a natural way an inner product given by

$$
\begin{aligned}
\left\langle u_{i, j}, v_{i, j}\right\rangle_{h} & :=\Delta x \Delta y \sum_{j=0}^{M} \sum_{i=0}^{N}\left(\nabla_{x} u_{i, j} \cdot \nabla_{x} v_{i, j}\right) \\
& +\Delta x \Delta y \sum_{j=0}^{M} \sum_{i=0}^{N}\left(\nabla_{y} u_{i, j} \cdot \nabla_{y} v_{i, j}\right)+\Delta x \Delta y \sum_{j=0}^{M} \sum_{i=0}^{N} q_{i, j} u_{i, j} v_{i, j},
\end{aligned}
$$

with associated norm

$$
\left\|u_{i, j}\right\|_{h}=\left(\left\|u_{i, j}\right\|^{2}+\Delta x \Delta y \sum_{j=0}^{M} \sum_{i=0}^{N} q_{i, j}\left|u_{i, j}\right|^{2}\right)^{1 / 2} .
$$

We are now able to introduce the discrete functional

$$
\begin{aligned}
J_{d}: l_{0}^{2}\left(\Omega_{d}\right) & \longrightarrow \mathbb{R} \\
u_{i, j} & \mapsto J_{d}\left(u_{i, j}\right)
\end{aligned}
$$

given explicitly by

$$
\begin{aligned}
J_{d}\left(u_{i, j}\right) & :=\frac{\Delta x \Delta y}{2} \sum_{i=0}^{N} \sum_{j=0}^{M}\left|\nabla_{x} u_{i, j}\right|^{2}+\frac{\Delta x \Delta y}{2} \sum_{j=0}^{N} \sum_{i=0}^{M}\left|\nabla_{y} u_{i, j}\right|^{2} \\
& +\frac{\Delta x \Delta y}{2} \sum_{i=0}^{N} \sum_{j=0}^{M} q_{i, j}\left|u_{i, j}\right|^{2}-\Delta x \Delta y \sum_{i=0}^{N} \sum_{j=0}^{M} f_{i, j} u_{i, j},
\end{aligned}
$$


and it obeys the following estimate:

$$
\begin{aligned}
\left|J_{d}\left(u_{i, j}\right)\right| & \leq \frac{\Delta x \Delta y}{2} \sum_{i=0}^{N} \sum_{j=0}^{M}\left|\nabla_{x} u_{i, j}\right|^{2}+\frac{\Delta x \Delta y}{2} \sum_{j=0}^{N} \sum_{i=0}^{M}\left|\nabla_{y} u_{i, j}\right|^{2} \\
& +\frac{\Delta x \Delta y}{2} \sum_{i=0}^{N} \sum_{j=0}^{M}\left|q_{i, j}\right|\left|u_{i, j}\right|^{2}+\Delta x \Delta y \sum_{i=0}^{N} \sum_{j=0}^{M}\left|f_{i, j}\right|\left|u_{i, j}\right| .
\end{aligned}
$$

Moreover, by using the inequality (see (3.7)), we obtain

$$
\left|J_{d}\left(u_{i, j}\right)\right| \leq \frac{1}{2}\left\|u_{i, j}\right\|_{h}^{2}+C\left\|u_{i, j}\right\|_{h}
$$

The first result of this article shows the existence of a unique discrete solution as a critical point of the functional (3.2), that is, if $u_{i, j} \in l_{0}^{2}\left(\Omega_{d}\right)$ is a critical point of this functional, then

$$
\delta\left(J_{d}\left(u_{i, j}\right)\right) v_{i, j}=0, \quad \forall v_{i, j} \in l_{0}^{2}\left(\Omega_{d}\right)
$$

where $\delta\left(J_{d}\right)$ denotes the derivative of $J_{d}$.

The next result allow us established a relationships between the norms $\left|u_{i, j}\right|_{l_{2}}^{2}$ and ||$u_{i, j} \|_{h}^{2}$.

Theorem 3.2 (Variational Characterization of the First Eigenvalue). Let $\Omega_{d}$ be a discrete set of $\left[0, L_{1}\right] \times\left[0, L_{2}\right]$ and $q_{i, j} \in l_{0}^{\infty}$. Define the functional $Q_{d}\left(u_{i, j}\right): l_{0}^{\infty}\left(\Omega_{d}\right) \backslash\{0\} \rightarrow \Omega_{d}$ as

$$
Q_{d}\left(u_{i, j}\right):=\frac{\sum_{i=0}^{N} \sum_{j=0}^{M}\left[\left|\nabla_{x} u_{i, j}\right|^{2}+\left|\nabla_{y} u_{i, j}\right|^{2}\right]+\sum_{i=0}^{N} \sum_{j=0}^{M} q_{i, j}\left|u_{i, j}\right|^{2}}{\sum_{i=0}^{N} \sum_{j=0}^{M}\left|u_{i, j}\right|^{2}} .
$$

Then, this functional (Rayleigh Quotient) obeys the following properties:

1. $\min _{u \in l_{0}^{\infty}\left(\Omega_{d}\right) \backslash\{0\}} Q_{d}\left(u_{i, j}\right)=\lambda_{1}$;

2. $Q_{d}\left(u_{i, j}\right)=\lambda_{1}$ if, and only if, $u_{i, j}$ is a weak solution of

$$
\widehat{P}_{d}:\left\{\begin{array}{l}
\Delta_{d} u_{i, j}+q_{i, j} u_{i, j}=\lambda_{1} u_{i, j}, 0 \leq i \leq N, 0 \leq j \leq M, \text { in } \Omega_{d}, \\
u_{i, 0}=u_{0, j}=0, \text { for all } 0 \leq i \leq N+1,0 \leq j \leq M+1 .
\end{array}\right.
$$

3. Every nontrivial solution of $\widehat{P}_{d}$ has defined sign in $\Omega_{d}$. In particular, this solution is different of zero a.e. in $\partial \Omega_{d}$;

4. The set of solutions of $\widehat{P}_{d}$ is unidimensional. In this case we say that $\lambda_{1}$ is simple. 
Proof. The proof may be adapted on the approach from Evan's book (see page 366, Theorem 2).

Theorem 3.3. Let $u_{i, j}$ solution of discrete system (1.6), then there exists a positive constant $C$ such that

$$
\left|u_{i, j}\right|_{l_{2}}^{2} \leq C|| u_{i, j} \|_{h}^{2}
$$

Proof. Follows from variational characterization of the first eigenvalue that

$$
\lambda_{1}=\min _{u \in l_{0}^{\infty}\left(\Omega_{d}\right) \backslash\{0\}} Q_{d}\left(u_{i, j}\right) \leq Q_{d}\left(u_{i, j}\right)=\frac{\left\|u_{i, j}\right\|_{h}^{2}}{\left|u_{i, j}\right|_{l_{2}}^{2}} .
$$

Consequently, we arrive at

$$
\left|u_{i, j}\right|_{l_{2}}^{2} \leq \frac{1}{\lambda_{1}}\left\|u_{i, j}\right\|_{h}^{2}
$$

\section{PROOF OF THE THEOREMS 1.1 and 1.2}

In this section, we prove the Theorems 1.1 and 1.2.

\subsection{Proof of the do Theorem 1.1}

First of all we note that $h \in C^{2, \alpha}(\bar{\Omega})$ guarantees that $u \in C^{4}(\bar{\Omega})$ and then we have that

$$
\left\|D^{4} u\right\|_{L_{\infty}(\Omega)}=\sup _{(x, y) \in \Omega, p+q=4}\left|\frac{\partial^{4} u}{\partial x^{p} \partial y^{q}}(x, y)\right| .
$$

On the other hand, by using the Taylor's expansion we obtain

$$
\begin{aligned}
\Delta u\left(x_{i}, y_{j}\right) & =\frac{u\left(x_{i}+\Delta x, y_{j}\right)-2 u\left(x_{i}, y_{j}\right)+u\left(x_{i}-\Delta x, y_{j}\right)}{\Delta x^{2}} \\
& +\frac{u\left(x_{i}, y_{j}+\Delta y\right)-2 u\left(x_{i}, y_{j}\right)+u\left(x_{i}, y_{j}-\Delta y\right)}{\Delta y^{2}} \\
& -\frac{1}{12}\left(\frac{\partial^{4} u}{\partial x^{4}}\left(x_{i}, y_{j}\right) \Delta x^{2}+\frac{\partial^{4} u}{\partial y^{4}}\left(x_{i}, y_{j}\right) \Delta y^{2}\right)+\mathcal{O}\left(\Delta x^{2}+\Delta y^{2}\right),
\end{aligned}
$$

from where we have 


$$
\begin{aligned}
\mathcal{L} u\left(x_{i}, y_{j}\right):= & -\Delta u\left(x_{i}, y_{j}\right)+q\left(x_{i}, y_{j}\right) u\left(x_{i}, y_{j}\right) \\
= & -\frac{u\left(x_{i}+\Delta x, y_{j}\right)-2 u\left(x_{i}, y_{j}\right)+u\left(x_{i}-\Delta x, y_{j}\right)}{\Delta x^{2}} \\
& -\frac{u\left(x_{i}, y_{j}+\Delta y\right)-2 u\left(x_{i}, y_{j}\right)+u\left(x_{i}, y_{j}-\Delta y\right)}{\Delta y^{2}} \\
& +q\left(x_{i}, y_{j}\right) u\left(x_{i}, y_{j}\right)+\frac{1}{12}\left(\frac{\partial^{4} u}{\partial x^{4}}\left(x_{i}, y_{j}\right) \Delta x^{2}+\frac{\partial^{4} u}{\partial y^{4}}\left(x_{i}, y_{j}\right) \Delta y^{2}\right) \\
& +\mathcal{O}\left(\Delta x^{4}+\Delta y^{4}\right),
\end{aligned}
$$

and then

$$
\mathcal{L} u\left(x_{i}, y_{j}\right)=\mathcal{L}_{d} u\left(x_{i}, y_{j}\right)+\frac{1}{12}\left(\frac{\partial^{4} u}{\partial x^{4}}\left(x_{i}, y_{j}\right) \Delta x^{2}+\frac{\partial^{4} u}{\partial y^{4}}\left(x_{i}, y_{j}\right) \Delta y^{2}\right)+\mathcal{O}\left(\Delta x^{4}+\Delta y^{4}\right) .
$$

Now, taking into account that

$$
\mathcal{L} u\left(x_{i}, y_{j}\right)=f\left(x_{i}, y_{j}\right)=f_{i, j},
$$

we have

$\mathcal{L}_{d} u\left(x_{i}, y_{j}\right)=\mathcal{L} u\left(x_{i}, y_{j}\right)+\frac{1}{12}\left(\frac{\partial^{4} u}{\partial x^{4}}\left(x_{i}, y_{j}\right) \Delta x^{2}+\frac{\partial^{4} u}{\partial y^{4}}\left(x_{i}, y_{j}\right) \Delta y^{2}\right)+\mathcal{O}\left(\Delta x^{4}+\Delta y^{4}\right)$,

and then we obtain

$$
\mathcal{L}_{d} u\left(x_{i}, y_{j}\right)=f_{i, j}+\frac{1}{12}\left(\frac{\partial^{4} u}{\partial x^{4}}\left(x_{i}, y_{j}\right) \Delta x^{2}+\frac{\partial^{4} u}{\partial y^{4}}\left(x_{i}, y_{j}\right) \Delta y^{2}\right)+\mathcal{O}\left(\Delta x^{4}+\Delta y^{4}\right) .
$$

Subtracting side by side this equation from the discretized problem (1.6) we obtain

$$
\mathcal{L}_{d}\left(u\left(x_{i}, y_{j}\right)-u_{i, j}\right)=\frac{1}{12}\left(\frac{\partial^{4} u}{\partial x^{4}}\left(x_{i}, y_{j}\right) \Delta x^{2}+\frac{\partial^{4} u}{\partial y^{4}}\left(x_{i}, y_{j}\right) \Delta y^{2}\right)+\mathcal{O}\left(\Delta x^{4}+\Delta y^{4}\right),
$$

which implies

$$
\begin{aligned}
\left\|\mathcal{L}_{d}\left(u\left(x_{i}, y_{j}\right)-u_{i, j}\right)\right\|_{\infty} & \leq \frac{1}{12}\left\|D^{4} u\right\|_{L_{\infty}(\Omega)}\left(\Delta x^{2}+\Delta y^{2}\right)+\mathcal{O}\left(\Delta x^{4}+\Delta y^{4}\right) . \\
& \leq C\left\|D^{4} u\right\|_{L_{\infty}(\Omega)}\left(\Delta x^{2}+\Delta y^{2}\right) .
\end{aligned}
$$

Therefore, by using the priori estimate given in the Theorem 2.2 we conclude our proof.

\subsection{Proof of the Theorem 1.2}

We are now ready to prove Theorem 1.2. To prove our assertive we show that there exists a discrete rate of change of $J_{d}\left(u_{i, j}\right)$. Moreover, we show that $J_{d}\left(u_{i, j}\right)$ is strictly convex and coercive at discrete setting of the finite difference method used here. 
Discrete rate: Let us consider the discrete functional to the discrete problem (1.6):

$$
\begin{aligned}
J_{d}\left(u_{i, j}\right) & =\frac{\Delta x \Delta y}{2} \sum_{i=0}^{N} \sum_{j=0}^{M}\left|\nabla_{x} u_{i, j}\right|^{2}+\frac{\Delta x \Delta y}{2} \sum_{j=0}^{N} \sum_{i=0}^{M}\left|\nabla_{y} u_{i, j}\right|^{2} \\
& +\frac{\Delta x \Delta y}{2} \sum_{i=0}^{N} \sum_{j=0}^{M} q_{i, j}\left|u_{i, j}\right|^{2}-\Delta x \Delta y \sum_{i=0}^{N} \sum_{j=0}^{M} f_{i, j} u_{i, j} .
\end{aligned}
$$

Using the inner product (1.10) and the norm (3.1) it follows that

$$
J_{d}\left(u_{i, j}\right)=\frac{1}{2}\|u\|_{h}^{2}-\left(f_{i, j}, u_{i, j}\right)_{l^{2}} .
$$

We claim that $J_{d}\left(u_{i, j}\right)$ is defined from a bilinear and limited form in discrete setting. Indeed, considering that

$$
\begin{aligned}
a_{d}: \quad l_{0}^{2}\left(\Omega_{d}\right) \times l_{0}^{2}\left(\Omega_{d}\right) & \rightarrow \mathbb{R} \\
\left(u_{i, j}, v_{i, j}\right) & \mapsto a_{d}\left(u_{i, j}, v_{i, j}\right)
\end{aligned}
$$

where

$$
\begin{aligned}
a_{d}\left(u_{i, j}, v_{i, j}\right) & =\frac{\Delta x \Delta y}{2} \sum_{j=1}^{M} \sum_{i=1}^{N}\left(\nabla_{x} u_{i, j} \cdot \nabla_{x} v_{i, j}\right) \\
& +\frac{\Delta x \Delta y}{2} \sum_{j=1}^{M} \sum_{i=1}^{N}\left(\nabla_{y} u_{i, j} \cdot \nabla_{y} v_{i, j}\right) \\
& +\frac{\Delta x \Delta y}{2} \sum_{j=1}^{M} \sum_{i=1}^{N} q_{i, j} u_{i, j} v_{i, j} \\
& -\Delta x \Delta y \sum_{i=0}^{N} \sum_{j=0}^{M} f_{i, j} u_{i, j}, \quad \forall u_{i, j}, v_{i, j} l_{0}^{2}\left(\Omega_{d}\right),
\end{aligned}
$$

it is immediate that $a_{d}(\cdot, \cdot)$ is bilinear and then we take $J_{d}\left(u_{i, j}\right)=a_{d}\left(u_{i, j}, u_{i, j}\right)$. From this, we obtain an estimative for $J_{d}\left(u_{i, j}\right)$, i.e.,

$$
\left|J_{d}\left(u_{i, j}\right)\right| \leq \frac{1}{2}\|u\|_{h}^{2}+\left|\left(f_{i, j}, u_{i, j}\right)_{l^{2}}\right| \leq \frac{1}{2}\|u\|_{h}^{2}+C\|u\|_{h} .
$$


Now, we show the following:

$$
\begin{aligned}
J_{d}\left(u_{i, j}+w_{i, j}\right) & =\frac{1}{2}\left\langle u_{i, j}+w_{i, j}, u_{i, j}+w_{i, j}\right\rangle_{h}-\left(f_{i, j}, u_{i, j}+w_{i, j}\right)_{l^{2}}, \\
& =\frac{1}{2}\left\langle u_{i, j}, u_{i, j}\right\rangle_{h}+\left\langle u_{i, j}, w_{i, j}\right\rangle_{h}+\frac{1}{2}\left\langle w_{i, j}, w_{i, j}\right\rangle_{h} \\
& -\left(f_{i, j}, u_{i, j}\right)_{l^{2}}-\left(f_{i, j}, w_{i, j}\right)_{l^{2}}, \\
& =J_{d}\left(u_{i, j}\right)+\left\langle u_{i, j}, w_{i, j}\right\rangle_{h}+\frac{1}{2}\left\langle w_{i, j}, w_{i, j}\right\rangle_{h}-\left(f_{i, j}, w_{i, j}\right)_{l^{2}},
\end{aligned}
$$

and then

$$
J_{d}\left(u_{i, j}+w_{i, j}\right)-J_{d}\left(u_{i, j}\right)=\left\langle u_{i, j}, w_{i, j}\right\rangle_{h}+\frac{1}{2}\left\langle w_{i, j}, w_{i, j}\right\rangle_{h}-\left(f_{i, j}, w_{i, j}\right)_{l^{2}} .
$$

Now, taking $r\left(w_{i, j}\right)=\left\|w_{i, j}\right\|_{h}^{2}$ we have $\lim _{w_{i, j} \rightarrow 0} \frac{r\left(w_{i, j}\right)}{\left\|w_{i, j}\right\|_{h}} \rightarrow 0$ and then $J_{d}\left(u_{i, j}\right)$ obeys

$$
\begin{aligned}
\delta\left(J_{d}\left(u_{i, j}\right)\right) w_{i, j} & =\lim _{w_{i, j} \rightarrow 0} \frac{J_{d}\left(u_{i, j}+w_{i, j}\right)-J_{d}\left(w_{i, j}\right)}{\left\|w_{i, j}\right\|} \\
& =\left\langle u_{i, j}, w_{i, j}\right\rangle_{h}-\left(f_{i, j}, w_{i, j}\right)_{l^{2}} .
\end{aligned}
$$

showing that the discrete rate of $J_{d}$ is finite. This corresponds to the discrete version of the differentiability of $J$ in Fréchet sense (cf. [9]).

Strictly convex: We note that

$$
\left(\delta\left(J_{d}\left(u_{i, j}\right)\right)-\delta\left(J_{d}\left(w_{i, j}\right)\right)\right)\left(u_{i, j}-w_{i, j}\right)>0 .
$$

Indeed,

$$
\begin{aligned}
\left(\delta\left(J_{d}\left(u_{i, j}\right)\right)-\delta\left(J_{d}\left(w_{i, j}\right)\right)\right)\left(u_{i, j}-w_{i, j}\right) & =\delta\left(J_{d}\left(u_{i, j}\right)\right)\left(u_{i, j}-w_{i, j}\right) \\
& -\delta\left(J_{d}\left(w_{i, j}\right)\right)\left(u_{i, j}-w_{i, j}\right) \\
& =\left\langle u_{i, j}-w_{i, j}, u_{i, j}-w_{i, j}\right\rangle_{h} \\
& =\left\|u_{i, j}-w_{i, j}\right\|_{h}^{2} .
\end{aligned}
$$

Therefore,

$$
\left(\delta\left(J_{d}\left(u_{i, j}\right)\right)-\delta\left(J_{d}\left(w_{i, j}\right)\right)\right)\left(u_{i, j}-w_{i, j}\right) \geq\left\|u_{i, j}-w_{i, j}\right\|_{h}^{2}>0, \text { if } u_{i, j} \neq w_{i, j} .
$$


Coercivity: Taking into account that

$$
\begin{aligned}
J_{d}\left(u_{i, j}\right) & :=\frac{\Delta x \Delta y}{2} \sum_{i=0}^{N} \sum_{j=0}^{M}\left|\nabla_{x} u_{i, j}\right|^{2}+\frac{\Delta x \Delta y}{2} \sum_{j=0}^{N} \sum_{i=0}^{M}\left|\nabla_{y} u_{i, j}\right|^{2} \\
& +\frac{\Delta x \Delta y}{2} \sum_{i=0}^{N} \sum_{j=0}^{M} q_{i, j}\left|u_{i, j}\right|^{2}-\Delta x \Delta y \sum_{i=0}^{N} \sum_{j=0}^{M} f_{i, j} u_{i, j},
\end{aligned}
$$

and by using inequality (3.7), we get

$$
J_{d}\left(u_{i, j}\right) \geq \frac{1}{2}\left\|u_{i, j}\right\|_{h}^{2}-C\left\|u_{i, j}\right\|_{h}
$$

and thus $J_{d}\left(u_{i, j}\right)$ is coercive and we conclude the proof of the Theorem 1.2.

\section{NUMERICAL SIMULATIONS}

In this section, we present some numerical results using the finite difference (1.6). Our goal is to show, by means of numerical experiments the results set out in the previous sections. This scheme results in a system of coupled algebraic equations that must be solved simultaneously. In matrix notation, the system can be written as

$$
A U=F,
$$

where $U$ represents the vector of unknowns, $F$ the vector of independent terms and $A$ the matrix of the system. It is important to say that the boundary conditions are to be applied before solving the system (5.1).

Our computational experiments were performed using MatLab considering $L_{1}=L_{2}=1, \Delta x=$ $\Delta y$, where we have adopted two partitions with 50 divisions in each of the directions $x$ and $y$, the one that gave us a mesh with 2500 points. Below we present numerical simulations.

\subsection{Discrete maximum principle simulations}

The experiment shown here were obtained considering two separate cases. In the first case (Figures 1 and 2), the simulations were made using $q_{i, j}=7 e^{-\left(2 x_{i}+3 y_{i}\right)}$ and $f_{i, j}=-1$. For the second case (Figures 3 and 4), the simulations were made using $q_{i, j}=7 \sin \left(x_{i}+3 y_{i}\right)$ and $f_{i, j}=1$.

Comments: They show us that the explicit finite difference numerical scheme (1.6) is robust enough to reproduce the results of our analysis, as in Figures 1 and 3 we observe that the maximum (minimum) value is reached at the border, such as the Discrete Maximum Principle. On the other hand, Figures 2 and 4 show us that functional energy is coercive and, therefore, inferiorly limited. 


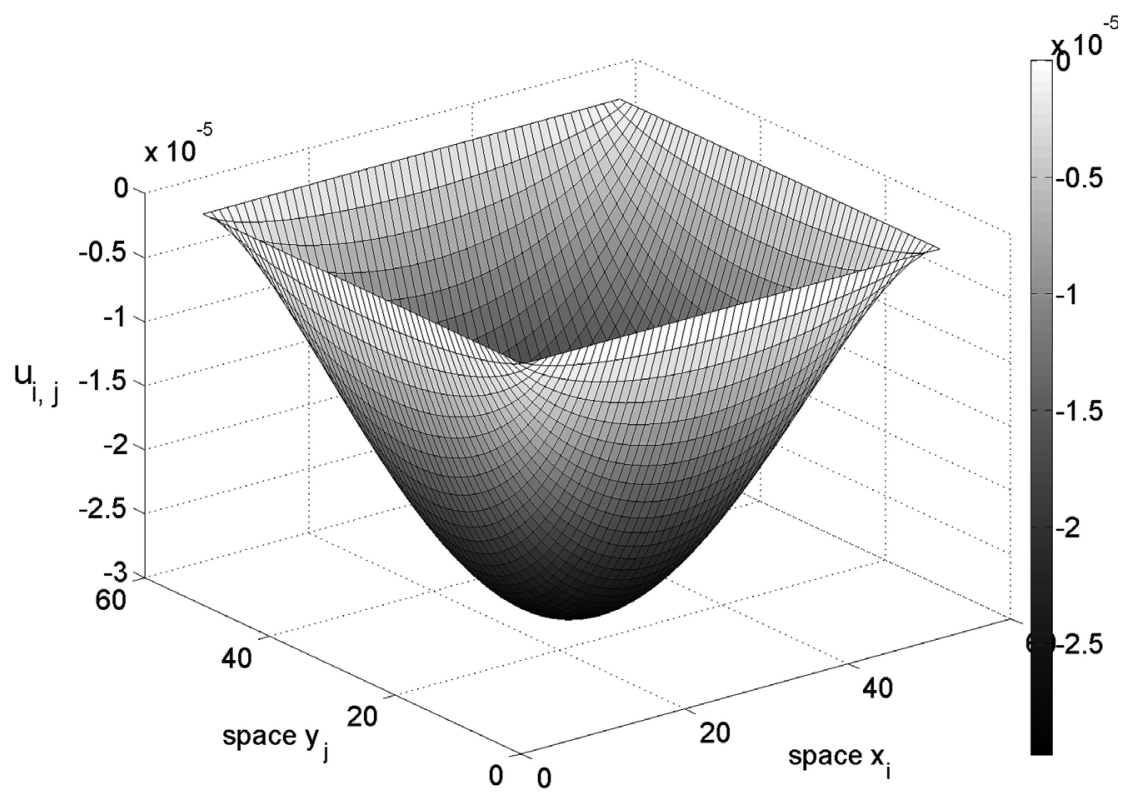

Figure 1: Numerical solution.

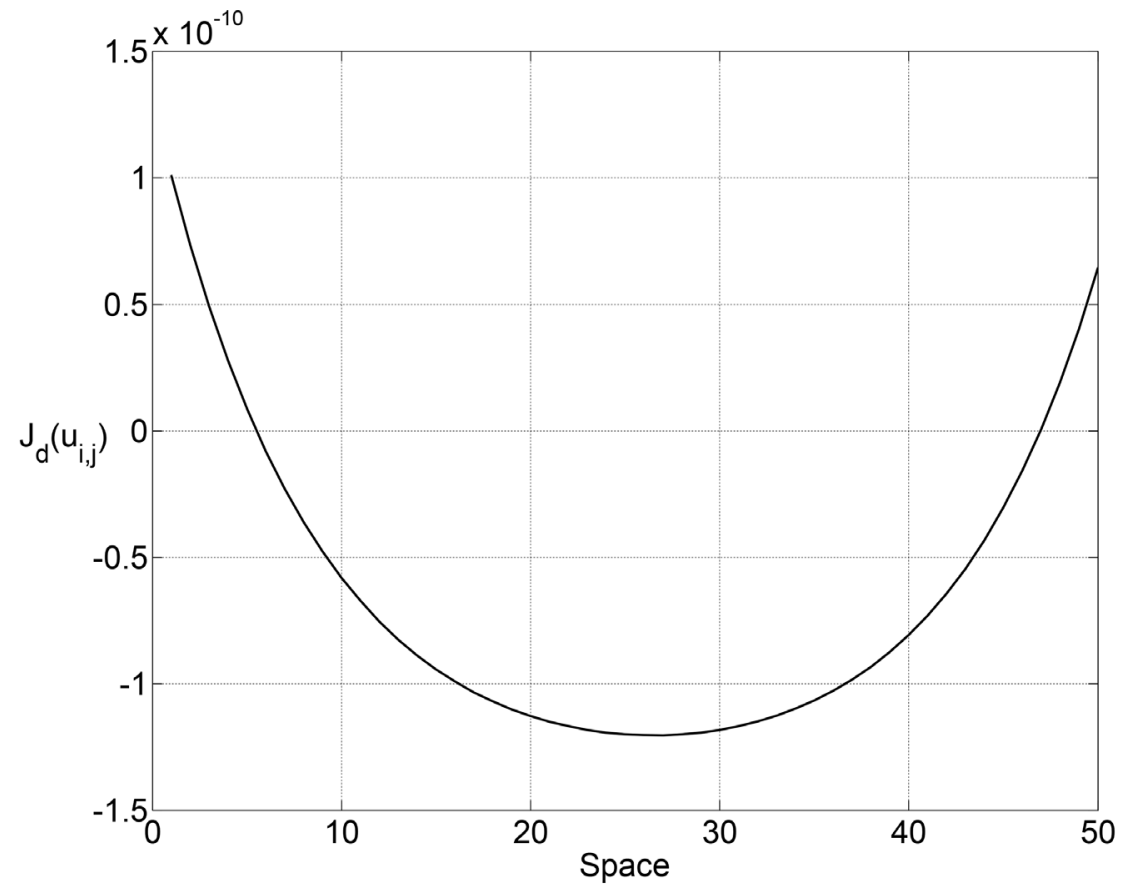

Figure 2: Functional energy. 


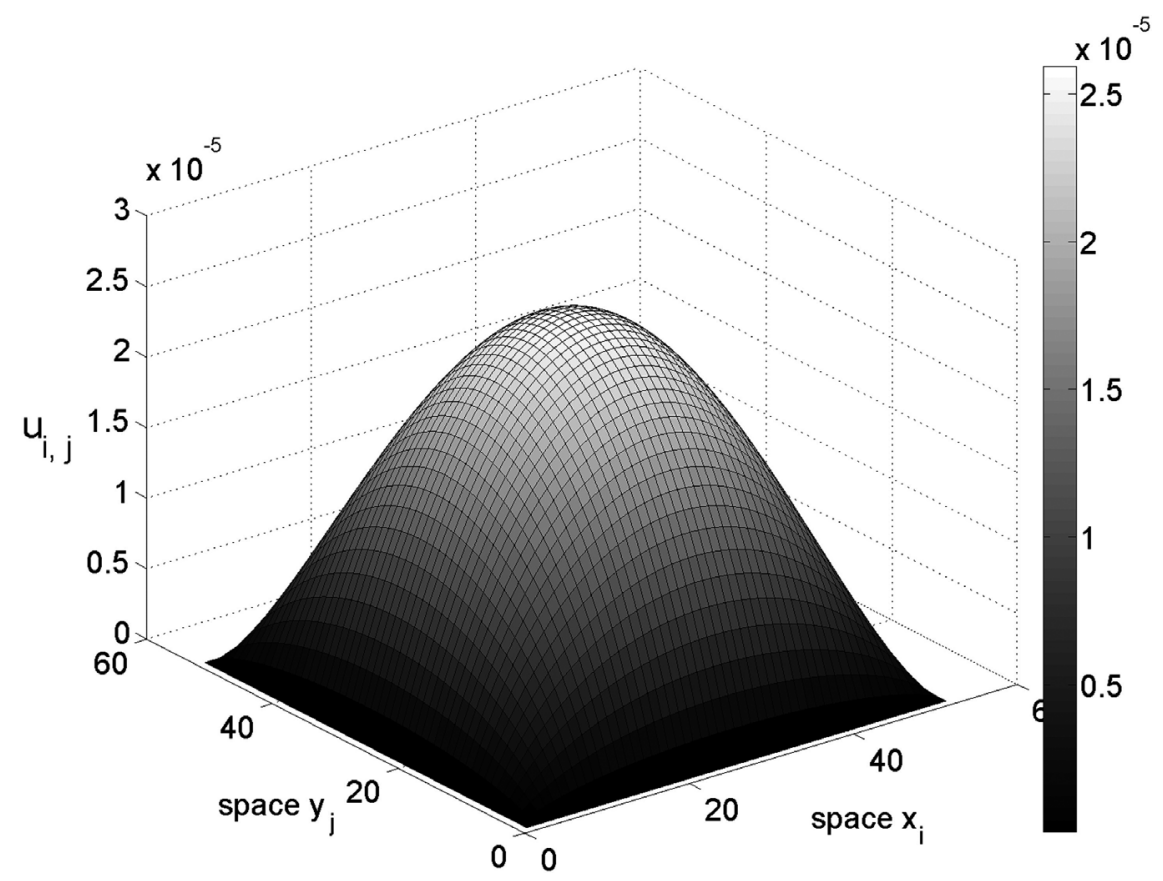

Figure 3: Numerical solution.

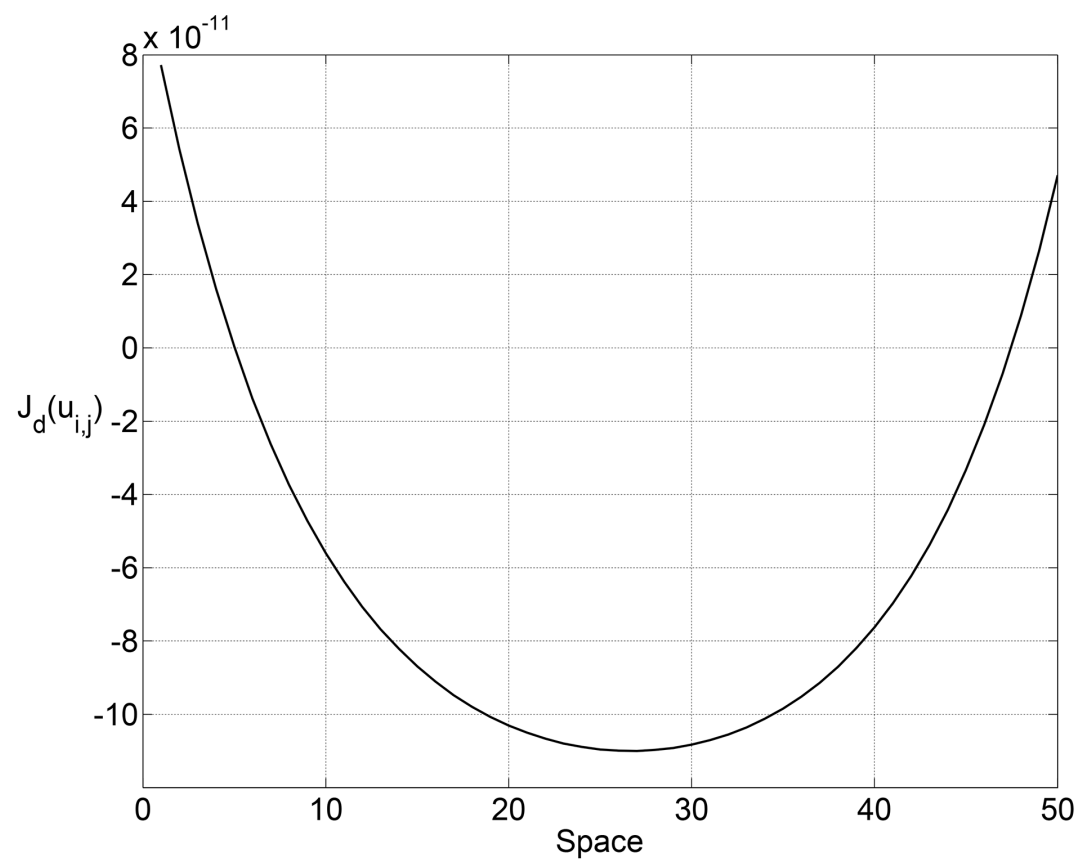

Figure 4: Functional energy. 
RESUMO. Neste artigo tratamos as questões de convergência, existência e unicidade da solução numérica de um problema elíptico discretizado pelo método de diferenças finitas. A fim de provarmos a existência e unicidade da solução numérica, usaremos uma configuração variacional adequada (em nível discreto) para garantirmos a existência de uma solução numérica pelo método de diferenças finitas.

Palavras-chave: equações elípticas, diferenças finitas, solvabilidade de soluções numéricas.

\section{REFERENCES}

[1] H. Brezis. Functional Analysis, Sobolev Spaces and Partial Differential Equations, Universitext, Springer, (2010).

[2] L. Evans. Partial Differential Equations, Graduate Studies in Mathematics, American Mathematical Society, 19 (1998), 749 pp.

[3] D. Gilbarg \& N.S. Trudinger. Elliptic Partial Differential Equations of Second Order, Classics in Mathematics, Springer, (2015).

[4] Q. Han \& F. Lin. Elliptic Partial Differential Equations, Courant Lecture Notes in Mathematics, American Mathematical Society, (2011).

[5] K.W. Morton \& D.F. Mayers. Numerical Solution of Partial Differential Equations: an Introduction, Cambridge University Press, (2005).

[6] J.W. Thomas. Numerical Partial Differential Equations: Conservation Laws and Elliptic Equations, Texts in Applied Mathematics, Springer, (1999).

[7] J.C. Strikwerda. Finite Difference Schemes and Partial Differential Equations, SIAM, (2004).

[8] W. Hackbusch. Elliptic Differential Equations: Theory and Numerical Treatment, Series in Computational Mathematics. Springer, 18 (1992), 311 pp.

[9] D. Behmardi \& E.D. Nayeri. Introduction of Frechet and Gateaux Derivative, Applied Mathematical Sciences, 2 (2008), 975-980. 